Table 2. US, AT \& confirmed diagnosis

\begin{tabular}{|c|c|c|c|c|c|c|}
\hline \multirow[t]{2}{*}{ Category } & \multicolumn{3}{|c|}{ Ultrasound } & \multirow[t]{2}{*}{ No of AT } & \multirow[t]{2}{*}{ Type of AT } & Final Diagnosis \\
\hline & +ve & Not done & -ve & & & \\
\hline LC (78) & 1 & 39 & 38 & 7 & 1x TAB $(-)$, СTB $(-)$ & Fibromyalgia \\
\hline & & & & & 1x TAB (-), MRA (-), MR neck (+) & Tongue cancer \\
\hline & & & & & $1 \times$ CTA $(+)$ & Stroke \\
\hline & & & & & 1x CTCAP (-) & IA \\
\hline & & & & & $1 \times$ PET (-) & PMR \\
\hline & & & & & $1 \times \mathrm{TAB}(-)$ & NA AION \\
\hline & & & & & 1x PET $(-)$ & CVA \\
\hline \multirow[t]{2}{*}{ IC (65) } & 30 & 3 & 32 & 15 & $5 \times$ TAB (-), 2x PET (-) & Not GCA \\
\hline & & & & & 2x TAB $(+), 6 x$ PET $(+)$ & GCA \\
\hline \multirow[t]{9}{*}{ HC (31) } & 25 & 0 & 6 & 10 & 1x PET (-) & URTI \\
\hline & & & & & $1 \times$ TAB (-) & NAAION \\
\hline & & & & & $2 \times$ PET $(+)$ & \\
\hline & & & & & $1 \times \operatorname{TAB}(+)$ & \\
\hline & & & & & $1 \times$ CTA $(+)$ & \\
\hline & & & & & 1x MRA (+) & GCA \\
\hline & & & & & 1x PET (-) & \\
\hline & & & & & $2 \times$ CTA $(-)$ & \\
\hline & & & & & 1x CTCAP (-) & \\
\hline
\end{tabular}

Abbreviations: AT, Additional test; CTA, Computed tomography angiogram; CTB, Computed tomography of brain; CTCAP, Computed tomography of chest, abdomen and pelvis; GCA, Giant cell arteritis; IA, Inflammatory arthritis; MRA, Magnetic resonant angiogram; NA AION, Non arteritic anterior ischemic optic neuritis; PET, Position emission tomography; TAB, Temporal artery biopsy; URTI, Upper respiratory tract infection

In IC, 30/65 (46\%) were US +ve 8 had AT (all GCA confirmed) while on treatment. $32(49 \%)$ US negative where 7 had AT (all GCA excluded). 3 did not have US. Sensitivity, specificity, accuracy of US was all $100 \%$, GCA prevalence $46 \%$, mean CRP 39.05 (SEM+/- 5.04)

US test performance overall sensitivity $95 \%$, specificity $98 \%$, accuracy $97 \%$ Conclusion: PTPS successfully stratifies GCA, excludes mimics and enhances US performance. The algorithm interprets correctly US findings and choice of AT.

References:

[1] Patil et al Clin Exp Rheumatol 2015;33(Suppl 89): S103-6.

[2] Laskou et al. Clin Exp Rheumatol. 2019 Feb 15

Figure-1: Probability based diagnostic algorithm for suspected GCA

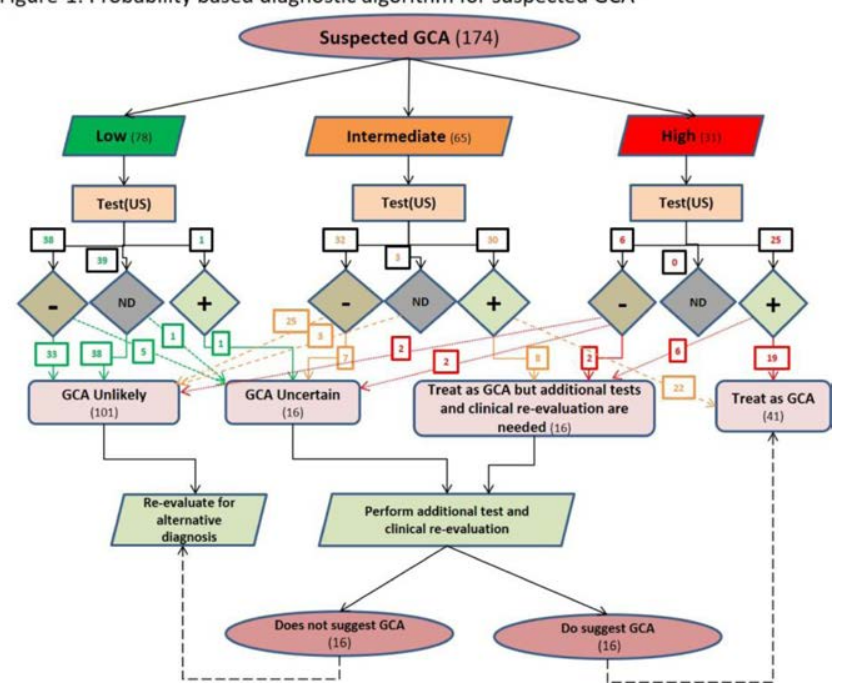

Disclosure of Interests: Alwin Sebastian: None declared, Abdul Kayani: None declared, Chavini Ranasinghe: None declared, Bhaskar Dasgupta Grant/research support from: Roche, Consultant of: Roche, Sanofi, GSK, BMS, AbbVie, Speakers bureau: Roche

DOI: 10.1136/annrheumdis-2020-eular.904

\section{SAT0250 \\ CLINICAL CHARACTERISTICS AND THE DISEASE ACTIVITY OF BEHCET'S DISEASE IN CHINA: A STUDY BASED ON SMART SYSTEM OF DISEASE MANAGEMENT (SSDM)}

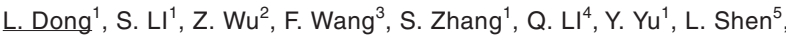
L. Luo ${ }^{6}$, P. Ji ${ }^{6}$, W. Liu ${ }^{7}$, T. Wang ${ }^{8}$, H. Xiao ${ }^{9}$, S. Jia ${ }^{9}$, Y. Jia ${ }^{9}$, F. Xiao ${ }^{9}$, L. Wu ${ }^{10}$ on behalf of SSDM Collaboration Group, China. ${ }^{1}$ Tongji Hospital Affiliated to Tongji Medical College of HUST, Wuhan, China; ${ }^{2}$ The First Affiliated Hospital of the Air Force Medical University, Xi'an, China; ${ }^{3}$ First Affiliated Hospital of Medical University of Anhui, Anhui, China; ${ }^{4}$ The First People's Hospital of Yunnan Province, Kunming, China; ${ }^{5}$ Union Hospital
Affiliated Tongji Medical College of HUST, Wuhan, China; ${ }^{6}$ The First Affiliated Hospital of Xinjiang Medical University, Urumqi, China; ${ }^{7}$ The First Teaching Hospital of Tianjin University of TCM, Tianjin, China; ${ }^{8}$ Beijing Anzhen Hospital, Capital Medical University, Beijing, China; ${ }^{9}$ Shanghai Gothic Internet Technology Co., Ltd., Shanghai, China; ${ }^{10}$ People's Hospital of Xinjiang Uygur Autonomous Region, Urumqi, China

Background: Behcet's disease (BD) is a systemic autoimmune disease that affects multiple organ systems with recurrent oral ulcers, genital ulcers and skin lesions. Behcet's Disease Current Activity Form (BDCAF) and Electronic Medical Record-based Activity Index (EMRAI) are commonly used internationally to evaluate the disease activity of $\mathrm{BD}$.

Objectives: This study aimed to analyze the clinical characteristics, the level of disease activity, and the incidence of anxiety and depression for Chinese BD patients. Patients can perform self-management of disease with SSDM.

Methods: SSDM is a series of doctor-patient interactive applications for self-management of patients with chronic diseases. Patients can perform self-assessment with SSDM and upload the data to their authorized doctors. The SSDM patients application system integrates the BDCAF and EMRAI into one scoring system. Patients could obtain scores of BDCAF and EMRAI by responding to one questionnaire through SSDM.

Results: From Apr 2017 to Jan 2020, 719 BD patients from 166 hospitals used SSDM, with a mean age of $38.97 \pm 12.71$ (14 81) years old, and median disease duration of 20.8 months. 719 patients performed BDCAF and EMRAI self-assessment 1321 times, 252 patients repeat assessments for 855 times. The mean score of BDCAF and EMRAI are $3.57 \pm 2.17$ and $3.44 \pm 1.90$, respectively. The matching degree of the two score was 0.8747 .

The most common clinical characteristics were oral ulcers $(83.73 \%)$, ocular symptoms $(62.03 \%)$, joint pain $(50.07 \%)$. The comparative study between males and females revealed significant difference in the aspects of epididymitis $(10.94 \%$ vs $0, p<0.001)$, genital ulcer $(35.09 \%$ vs $44.93 \%, p=0.01)$, headache $(24.15 \%$ vs $33.92 \%, p=0.01)$ and superficial thrombophlebitis $(24.15 \%$ vs $33.92 \%, p=0.01)$ Table 1.

Table 1. Clinical Characteristics of different systems in Chinese BD patients.

\begin{tabular}{lcccc}
\hline Presence of clinical characteristics & Total & Males & Females & P value \\
\hline Oral ulcer & $83.73 \%(602)$ & $86.42 \%(229)$ & $82.16 \%(373)$ & 0.14 \\
Genital ulcer & $41.31 \%(297)$ & $35.09 \%(93)$ & $44.93 \%(204)$ & $0.01^{*}$ \\
Epididymitis & $4.03 \%(29)$ & $10.94 \%(29)$ & $0(0)$ & $<0.001^{\text {** }}$ \\
Erythema & $29.49 \%(212)$ & $29.43 \%(78)$ & $29.52 \%(134)$ & 0.98 \\
Skin lesions & $26.84 \%(193)$ & $28.68 \%(76)$ & $25.77 \%(117)$ & 0.4 \\
Superficial thrombophlebitis & $30.32 \%(218)$ & $24.15 \%(64)$ & $33.92 \%(154)$ & $0.01^{*}$ \\
Headache & $30.32 \%(218)$ & $24.15 \%(64)$ & $33.92 \%(154)$ & $0.01^{*}$ \\
Joint pain & $50.07 \%(360)$ & $51.32 \%(136)$ & $49.34 \%(224)$ & 0.61 \\
Arthritis & $14.60 \%(105)$ & $14.72 \%(39)$ & $14.54 \%(66)$ & 0.95 \\
Gastrointestinal involvement & $24.90 \%(179)$ & $27.92 \%(74)$ & $23.13 \%(105)$ & 0.15 \\
Ocular symptoms & $62.03 \%(446)$ & $62.64 \%(166)$ & $61.67 \%(180)$ & 0.79 \\
Nervous involvement & $23.78 \%(171)$ & $25.66 \%(68)$ & $22.69 \%(103)$ & 0.37 \\
Vascular involvement & $15.72 \%(113)$ & $18.11 \%(48)$ & $14.32 \%(65)$ & 0.18
\end{tabular}

${ }^{*} \mathrm{P}$ values are for the comparison between the males and females.

Conclusion: Chinese BD patients can effectively perform BDCAF and EMRA self-assessment with SSDM. The results of the assessment conducted by the two scoring systems are similar. The clinical characteristics of Chinese BD were different depending on gender.

Acknowledgments: : Smart system of disease management (SSDM) was developed by Shanghai Gothic Internet Technology Co., Ltd.

Disclosure of Interests: None declared

DOI: 10.1136/annrheumdis-2020-eular.1470

\section{SAT0251 ANGIOGRAPHIC PROGRESSION OF DISEASE IN LARGE-VESSEL VASCULITIS}

K. A. Quinn ${ }^{1,2}$, H. Dashora ${ }^{1}$, M. Ahlman ${ }^{2}$, P. Grayson ${ }^{1} .{ }^{1}$ National Institutes of Health, Bethesda, United States of America; ${ }^{2}$ Georgetown University Hospital, Washington, DC, United States of America

Background: Angiography is essential to detect vascular disease in patients with large-vessel vasculitis (LVV). Guidelines differ on the role of periodic angiography to monitor patients with LVV, in part because there is limited prospective data characterizing the natural history of angiographic disease. Development of new areas of arterial damage, even in periods of apparent clinical remission, has been reported in LVV; however, whether this is a common or rare phenomenon is unknown.

Objectives: To characterize angiographic progression of disease over time in Takayasu's arteritis (TAK) compared to giant cell arteritis (GCA).

Methods: Patients with GCA or TAK were recruited into a prospective, observational cohort. Patients fulfilled the 1990 American College of Rheumatology (ACR) Classification Criteria for TAK or modified 1990 ACR Criteria for GCA 